\title{
Prevalence and prognostic value of various types of right ventricular dysfunction in mechanically ventilated septic patients
}

Hongmin Zhang ${ }^{1}$, Wei Huang ${ }^{1}$, Qing Zhang ${ }^{1}$, Xiukai Chen ${ }^{2}$, Xiaoting Wang ${ }^{1}$ and Dawei Liu ${ }^{1 *}$ Critical Care

Ultrasound Study Group

\begin{abstract}
Introduction: Right ventricle (RV) dilation in combination with elevated central venous pressure (CVP), which is a state of RV congestion, is seen as a sign of RV failure (RVF). On the other hand, RV systolic function is usually assessed by tricuspid annular plane systolic excursion (TAPSE) and fractional area change (FAC). This study aimed to investigate the prevalence and prognostic value of RVF and RV systolic dysfunction (RVSD) in septic patients.

Methods: Mechanically ventilated sepsis and septic shock patients were included. We collected haemodynamic and echocardiographic parameters as well as prognostic information including mechanical ventilation duration, length of ICU stay and 30-day mortality. RVF was defined as a right and left ventricular end-diastolic area ratio $\geq 0.6$ in combination with CVP $\geq 8 \mathrm{mmHg}$. RVSD was defined as TAPSE $<16 \mathrm{~mm}$ or FAC $<35 \%$.

Results: A total of 215 patients were enrolled in this study, and the patients were divided into 4 groups: patients with normal RV function (normal, $n=101$ ), patients with RVF but without RVSD (RVF only, $n=38$ ), patients with RVSD but without RVF (RVSD only, $n=44$ ), and patients with combined RVF-RVSD (RVF/RVSD, $n=32$ ). The RVF/RVSD group and RVSD only group had a lower cardiac index than the RVF only group and normal groups $(p<0.05)$. At 30 days after ICU admission, $50.0 \%$ of patients had died in the RVF/RVSD group, which was much higher than the mortality in the RVF only group (13.2\%) and normal group (13.9\%) $(p<0.05)$. In a Cox regression analysis, the presence of RVF/RVSD was independently associated with 30-day mortality ( $H R 3.004$, 95\% Cl:1.370-6.587, $p=0.006$ ). In contrast, neither the presence of RVF only nor the presence of RVSD only was associated with 30-day mortality (HR 0.951, 95\% Cl:0.305$2.960, p=0.931 ;$ HR $1.912,95 \%$ Cl:0.853-4.287, $p=0.116$, respectively).
\end{abstract}

Conclusion: The presence of combined RVF-RVSD was associated with 30-day mortality in mechanically ventilated septic patients. Additional studies are needed to confirm and expand this finding.

Keywords: Right ventricular failure, Right ventricular systolic dysfunction, Sepsis, Prognosis

\footnotetext{
*Correspondence: dwliu2015@sina.com

${ }^{1}$ Department of Critical Care Medicine, Peking Union Medical College Hospital, Chinese Academy of Medical Sciences and Peking Union Medical College, 1\# Shuai Fu Yuan, Dong Cheng District, Beijing 100730, China

Full list of author information is available at the end of the article
}

\section{Introduction}

Sepsis is a major public concern and the leading cause of mortality in critically ill patients $[1,2]$. Myocardial dysfunction is common in sepsis patients and can involve the left ventricle (LV) as well as the right ventricle (RV) $[3,4]$. Unlike the LV, the geometry of the RV is complex, and RV longitudinal strain and 3D echo are not readily available in the intensive care unit (ICU) [5]. Thus, tricuspid annular plane systolic excursion (TAPSE), and 
fractional area change (FAC) remain the most commonly used quantitative parameters of RV systolic function [6, 7].

RV dilation is usually represented by the ratio of RV and LV end-diastolic areas [8, 9]. Vieillard-Baron and his colleagues contended that RV dilation in combination with elevated central venous pressure (CVP) was a state of RV congestion and could unmask the occurrence of RV failure (RVF). They reported that RVF was more sensitive than TAPSE in the assessment of volume responsiveness in septic shock patients [10]. Prior studies have proven that RV dysfunction is associated with long-term prognosis in septic patients [11-13]. However, whether RVF, diagnosed by RV dilation and elevated CVP, was also of prognostic value has not been reported. Therefore, we performed this study to investigate the prevalence of RVF and RV systolic dysfunction (RVSD) and their association with cardiac output, ICU stay and 30-day mortality in mechanically ventilated septic patients.

\section{Patients and methods}

\section{Study population}

This study was an observational study conducted at a tertiary hospital's intensive care unit (ICU). We retrospectively studied a cohort of adult septic patients who were on mechanical ventilation from 1 May 2018 to 1 August 2020.

We adopted the same definition of sepsis and septic shock as described in Sepsis-3 [14]. The exclusion criteria included the following: lack of CVP monitoring; lack of MV support via tracheal intubation; intra-abdominal pressure above $12 \mathrm{mmHg}$; new onset of acute coronary syndrome within 1 week; severe valvular disease or history of valvular surgery; history of chronic pulmonary hypertension; insufficient echocardiographic image; and withholding of life support.

\section{Echocardiography}

Echocardiograms were recorded within the first $24 \mathrm{~h}$ of ICU admission. Two physicians (H Zhang and Q Zhang) with 10 years of echo experience obtained the images and they were blinded to the clinical states of the patients upon echo examination. The echo results were reported based on the PRICES statement [15]. At least three cardiac cycles were analysed and averaged. M-mode and Doppler echocardiographic measurements were taken according to standard protocols. The measurement of TAPSE, left ventricular ejection fraction (LVEF), averaged tissue Doppler velocity of lateral and medial mitral annuli at early diastole (e'), and tricuspid regurgitation (TR) were performed as previously described [12]. The ratio of RV end-diastolic area and LV end-diastolic area (R/LVEDA) was obtained at the end of ventricular diastole. FAC was defined as (end-diastolic area - endsystolic area)/end-diastolic area $\times 100$ [6]. The E velocity was measured using pulsed wave Doppler with the sample volume placed between the tips of the mitral valve. The diameter of the left ventricular outflow tract (LVOT) was obtained at the parasternal long-axis view. The velocity-time integral (VTI) was obtained by positioning the sample volume at the LVOT approximately $0.5 \mathrm{~cm}$ below the aortic valve via pulsed Doppler imaging [16]. Cardiac output $(\mathrm{CO})$ was calculated using the following formula: $\mathrm{CO}=\pi \times(\text { LVOT diameter } / 2)^{2} \times \mathrm{VTI} \times$ heart rate. The $\mathrm{CO}$ was then indexed to body surface area.

\section{Other parameters collected}

We collected the patients' demographic information, Acute Physiology and Chronic Health Evaluation (APACHE) II score, and Sequential Organ Failure Assessment (SOFA) score at ICU admission. Each patient's heart rate (HR), mean arterial pressure (MAP), CVP, positive end-expiratory pressure (PEEP), and plateau pressure (Pplat) were also collected at the time of the echo examination.

Definition RVF was defined as $R / L V E D A \geq 0.6$ in combination with CVP $\geq 8 \mathrm{mmHg}$ according to a recent study by Vieillard-Baron et al. [10]. RVSD was defined as TAPSE $<16 \mathrm{~mm}$ or FAC $<35 \%[6,17]$.

\section{Outcomes}

The primary outcome was 30-day survival, and the secondary outcomes included length of ICU stay, mechanical ventilation (MV) duration and cardiac index.

\section{Statistical analysis}

We performed statistical analysis using SPSS 13.0 (SPSS, Inc., Chicago, Illinois, USA). Continuous variables are expressed as the mean \pm SD or as the median and the interquartile range. Categorical variables are presented as frequencies and percentages. The distributions of the continuous values were assessed for normality by the Kolmogorov-Smirnov test. Differences among groups were assessed by one-way ANOVA, the Kruskal-Wallis test, the Chi-squared test, or Fisher's exact test, as appropriate. If necessary, a Dunn-Bonferroni test was performed for post hoc comparisons. Receiver operating characteristic (ROC) curves were generated and the areas under each respective curve were calculated. Prognostic factors for 30-day mortality were determined using the Cox regression model. The following variables were considered for the survival analysis: age, SOFA score, APACHE II score, PEEP, Pplat, LVEF, E/e', RVF and 
RVSD. The variables that had $p<0.1$ in the univariable model were included in the multivariable model and the hazard ratio was calculated, together with its $95 \%$ confidence interval. Cumulative survival curves of the 30-day follow-up were estimated with the KaplanMeier method. Sensitivity analyses were performed using different cut-off values for RVF, as well as incorporating LV systolic dysfunction, when investigating the association between RV function and 30-day mortality. Intraobserver and interobserver variabilities in LVEF, TR velocity and FAC were assessed in 20 randomly selected patients and were tested using intraclass correlation coefficients (ICCs). An ICC $>0.8$ was considered excellent agreement. Two-tailed $p<0.05$ was considered significant.

\section{Results}

\section{General characteristics}

In all, 368 patients were screened for enrolment, and 215 patients were included in this study. The patients were divided into 4 cohorts based on the presence of RVF and RVSD: patients with normal RV function (normal, $n=101$ ), patients with RVF but without RVSD (RVF only, $n=38$ ), patients with RVSD but without RVF (RVSD only, $n=44)$, and patients with combined RVF-RVSD (RVF/RVSD, $n=32$ ) (Fig. 1). The general characteristics are listed in Additional file 1: Tables S1 and S2.

The four groups had similar age and sex proportions. The RVF/RVSD group had higher APACHE II and SOFA scores than the RVF and normal groups $(p<0.05)$. The RVF/RVSD group had the highest PEEP level among all groups $(p=0.001)$ (Table 1$)$.

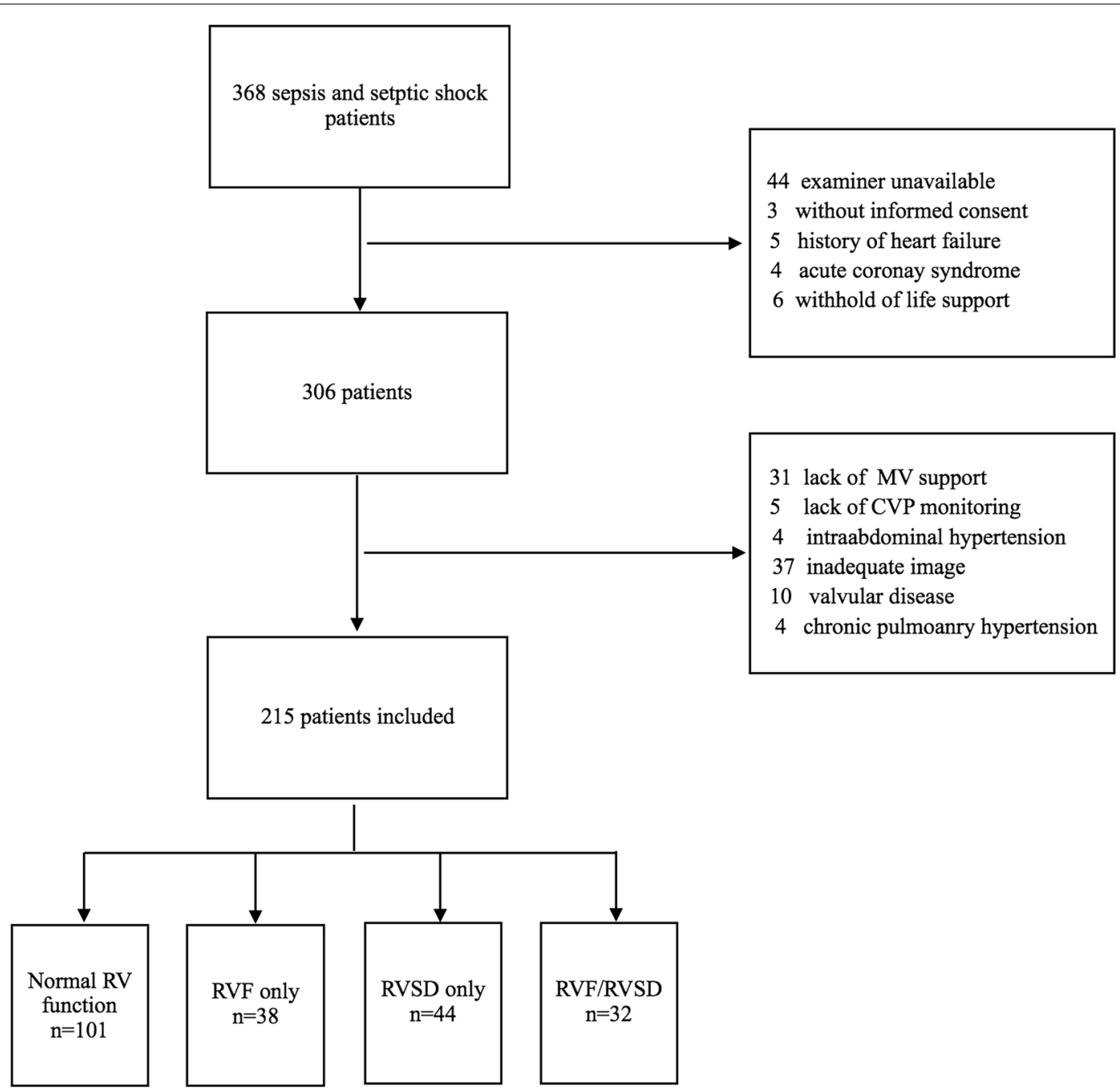

Fig. 1 Flowchart of the study. MV mechanical ventilation, CVP central venous pressure, $R V$ right ventricle, $R V F$ only patients with RV failure but without RV systolic dysfunction, RVSD only patients with RVSD but without RV failure; RVF/RVSD patients with combined RVF-RVSD 
Table 1 Demographics, illness severity, and haemodynamic and echocardiographic findings

\begin{tabular}{|c|c|c|c|c|c|c|}
\hline Categories & All patients $(n=215)$ & Normal $(n=101)$ & RVF only $(n=38)$ & RVSD only $(n=44)$ & RVF/RVSD $(n=32)$ & $p$ value \\
\hline Age (year) & $65(50,73)$ & $64(50,73)$ & $65(47,74)$ & $64(57,75)$ & $65(51,74)$ & 0.774 \\
\hline Sex (male, \%) & $134(62.3 \%)$ & $61(60.4 \%)$ & $2463.2 \%)$ & $28(63.6 \%)$ & $21(65.6 \%)$ & 0.104 \\
\hline APACHE ॥ & $20(15,26)$ & $19(13,26)$ & $19(14,24)$ & $22(17,27)$ & $24(18,30)$ & $0.043^{b, c}$ \\
\hline SOFA & $12(9,14)$ & $11(8,13)$ & $12(8,13)$ & $13(10,15)$ & $14(11,17)$ & $0.007^{b, c, e}$ \\
\hline HR (bpm) & $94 \pm 20$ & $93 \pm 16$ & $88 \pm 21$ & $98 \pm 22$ & $97 \pm 22$ & 0.109 \\
\hline MAP (mmHg) & $76(66,82)$ & $75(66,85)$ & $77(71,84)$ & $76(66,83)$ & $72(68,76)$ & 0.268 \\
\hline CVP $(\mathrm{mmHg})$ & $9(7,11)$ & $8(6,10)$ & $9(8,11)$ & $8(7,10)$ & $10(8,12)$ & $<0.001^{a, c, f}$ \\
\hline $\operatorname{PEEP}\left(\mathrm{cmH}_{2} \mathrm{O}\right)$ & $5(5,8)$ & $5(5,6)$ & $5(5,7)$ & $6(5,8)$ & $6(5,10)$ & $0.001^{b, c, e}$ \\
\hline Pplat $\left(\mathrm{cmH}_{2} \mathrm{O}\right)$ & $18(16,22)$ & $18(16,21)$ & $19(15,22)$ & $18(16,20)$ & $20(18,23)$ & 0.097 \\
\hline *Fluid before echo (ml) & $3764(3206,4589)$ & $3722(2677,4704)$ & $3701(3193,4471)$ & $3773(3326,4598)$ & $3884(2945,4804)$ & 0.992 \\
\hline R/LVEDA & $0.55(0.45,0.65)$ & $0.49(0.43,0.55)$ & $0.67(0.63,0.71)$ & $0.45(0.39,0.53)$ & $0.68(0.63,0.72)$ & $<0.001^{a, c, d, f}$ \\
\hline TAPSE (mm) & $19.0 \pm 5.1$ & $21.8 \pm 3.6$ & $21.5 \pm 4.0$ & $14.0 \pm 2.9$ & $14.0 \pm 3.3$ & $<0.001^{b, c, d, e}$ \\
\hline FAC (\%) & $46(38,52)$ & $49(44,55)$ & $48(41,55)$ & $34(29,47)$ & $32(29,44)$ & $<0.001^{b, c, d, e}$ \\
\hline $\mathrm{TR}(\mathrm{m} / \mathrm{s})$ & $2.4 \pm 0.5$ & $2.4 \pm 0.3$ & $2.4 \pm 0.4$ & $2.4 \pm 0.5$ & $2.6 \pm 0.5$ & 0.112 \\
\hline LVEF (\%) & $60(50,69)$ & $62(56,70)$ & $63(54,69)$ & $55(44,62)$ & $52(47,63)$ & $<0.001^{c, d, e}$ \\
\hline$E / e^{\prime}$ & $8.5(6.6,10.7)$ & $8.0(6.6,10.0)$ & $7.8(6.5,10.8)$ & $8.9(6.7,12.2)$ & $9.8(5.7,13.5)$ & 0.060 \\
\hline $\mathrm{Cl}\left(\mathrm{L} / \mathrm{min} / \mathrm{m}^{2}\right)$ & $3.4(2.8,4.0)$ & $3.6(3.0,4.2)$ & $3.4(2.9,4.3)$ & $3.1(2.5,3.8)$ & $3.1(2.7,3.7)$ & $<0.001^{c, d, e}$ \\
\hline MV duration (hr) & $100(36,235)$ & $91(30,232)$ & $93(30,172)$ & $105(67,211)$ & $138(62,282)$ & 0.379 \\
\hline ICU stay (day) & $6(3,12)$ & $6(3,11)$ & $4(3,10)$ & $7(4,12)$ & $7(3,14)$ & 0.086 \\
\hline 30-day mortality $(n, \%)$ & $50(23.2 \%)$ & $14(13.9 \%)$ & $5(13.2 \%)$ & 15 (34.1\%) & $16(50 \%)$ & $<0.001^{b, c}$ \\
\hline
\end{tabular}

*Fluid administered within $24 \mathrm{~h}$ before echo examination

${ }^{a}$ RVF/RVSD vs. RVSD, $p<0.05 ;{ }^{\text {B }}$ RVF/RVSD vs. RVF, $p<0.05 ;{ }^{C}$ RVF/RVSD vs. Normal, $p<0.05 ;{ }^{\mathrm{d}} \mathrm{RVSD}$ vs. RVF, $p<0.05 ;{ }^{\mathrm{e}} \mathrm{RVSD}$ vs. Normal, $p<0.05 ;{ }^{\mathrm{f}} \mathrm{RVF}$ vs. Normal, $p<0.05$ APACHE Acute Physiology and Chronic Health Evaluation, SOFA Sequential Organ Failure Assessment, HR heart rate, MAP: mean arterial pressure, CVP central venous pressure, PEEP positive end-expiratory pressure, Pplat plateau pressure, R/LVEDA ratio of right and left end-diastolic area, TAPSE tricuspid annular plane systolic excursion, FAC fractional area change, $T R$ tricuspid regurgitation, $L V E F$ left ventricular ejection fraction, $C I$ cardiac index, MV mechanical ventilation, ICU intensive care unit

\section{Comparison of haemodynamic and echocardiographic parameters}

The intraobserver variability analysis revealed that the ICCs for LVEF, TR velocity and FAC were 0.908 (95\% CI: 0.782-0.962), 0.916 (95\% CI: 0.801-0.966), and 0.851 (95\% CI: 0.661-0.938), respectively. The interobserver variabilities for LVEF, TR velocity and FAC were 0.875 (95\% CI: 0.712-0.949), 0.904 (95\% CI: 0.758-0.962), and 0.827 (95\% CI: 0.614-0.928), respectively.

The four groups had similar HR and MAP. Both the RVF/RVSD group and RVSD only groups had a lower LVEF than the normal group ( $p<0.05)$ (Table 1, Fig. 2e).

\section{Primary outcome}

At 30 days after ICU admission, $50.0 \%$ of patients had died in the RVF/RVSD group, which was much higher than the mortality in the RVF only group (13.2\%) and normal groups $(13.9 \%)(p<0.05)$. The mortality in the RVF/RVSD group was also higher than that in the RVSD group (34.1\%), but was not statistically significant.

The ROC analysis showed that the areas under the curve for CVP, R/LVEDA, TAPSE and FAC were 0.644 $(p=0.006) ; 0.525(p=0.634) ; 0.652(p=0.004)$ and $0.690(p<0.001)$, respectively (Fig. 3, Table 2$)$.

We generated Kaplan-Meier curves for estimated survival at 30 days after ICU admission. The RVF/RVSD group had higher mortality than the RVF only and normal groups (RVF/RVSD vs. RVF only, log-rank:12.613, $p<0.001 ; \quad$ RVF/RVSD vs. normal, log-rank:25.208, $p<0.001)$. The RVF/RVSD group also had higher mortality than the RVSD only group, but was not statistically significant (RVF/RVSD vs. RVSD only, logrank:3.662, $p=0.057$ ). The RVSD only group had higher mortality than the RVF only group and normal groups (RVSD only vs. RVF only, log-rank:3.995, $p=0.046$; RVSD only vs. normal, log-rank: 7.376, $p=0.007)$. No difference was found between the RVF only group and normal groups (RVF only vs. normal, log-rank: 0.012, $p=0.912$ ) (Fig. 4).

In a Cox regression survival analysis, after adjusting for APACHEII, SOFA, PEEP, Pplat, and E/e', the presence of RVF/RVSD was independently associated with 30-day mortality (HR 3.004, 95\% CI: 1.370-6.587, $p=0.006$ ). In contrast, neither the presence of RVF only nor the presence of RVSD only was associated with 30-day mortality 

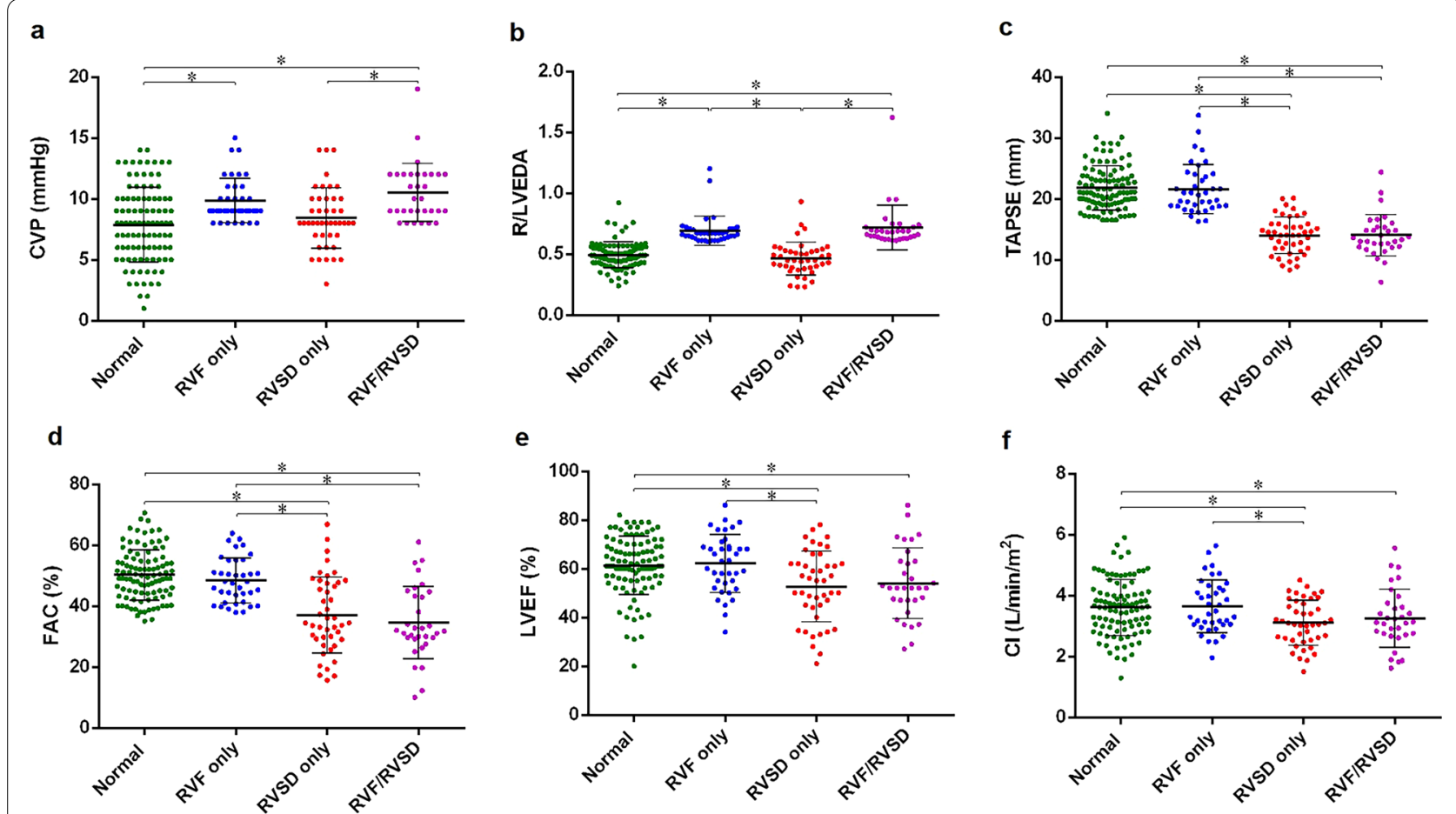

Fig. 2 Haemodynamic and echocardiographic parameters in four groups. a RVF/RVSD group had higher CVP than RVSD only group and normal group $(p<0.005)$. RVF only group had higher CVP than normal group $(p<0.005)$. b RVF/RVSD group and RVF only group had higher R/LVEDA ratio than RVSD only group and normal group ( $p<0.05)$. c RVF/RVSD group and RVSD only group had lower TAPSE than RVF only group and normal group $(p<0.05)$. d RVF/RVSD group and RVSD only group had lower FAC than RVF only group and normal group $(p<0.05)$. e RVF/RVSD group and RVSD only group had lower LVEF than normal group $(p<0.05)$. $\mathbf{R V F} /$ RVSD group and RVSD only group had lower CI than RVF only group and normal group $(p<0.05)$

(HR 0.951, 95\% CI: 0.305-2.960, $p=0.931 ;$ HR 1.912, 95\% CI:0.853-4.287, $p=0.116$ ) (Table 3).

\section{Sensitivity analysis}

We performed sensitivity analysis by using $\mathrm{CVP} \geq 10 \mathrm{mmHg}, \mathrm{CVP} \geq 12 \mathrm{mmHg}$, and R/LEDA $\geq 0.7$ separately as cut-off values of RVF, and found that RVF was still not an independent predictor of 30-day mortality in these patients.

\section{Secondary outcomes}

The RVF/RVSD and RVSD only groups had a lower cardiac index than the RVF only group and normal groups $(p<0.05)$. No significant difference was found regarding MV duration or length of ICU stay among the four groups (Table 1, Fig. 2f).

\section{Discussion}

In this study, we investigated the prevalence of RVF and RVSD and their association with short-term mortality in mechanically ventilated septic patients. We found that the presence of combined RVF-RVSD was associated with 30-day mortality. Neither RVF nor RVSD, when occurring alone, was a predictor of 30-day mortality in these patients.

The definition and criteria of RVF reported by Vieillard-Baron are reasonable, which provides physicians with a new perspective for RV function evaluation. An acute elevation in RV preload or afterload is manifested with RV dilation, which can be quickly estimated by R/ LVEDA [7, 8]. On the other hand, the primary function of the RV is to keep CVP as low as possible [18]. When the RV fails, CVP will rise inevitably. Therefore, the diagnosis of RVF based on RV dilation and CVP makes sense in the appraisal of RV function.

Although CVP was one of the criteria to define RVF, our results revealed that the CVP values among the four groups, given significant differences, were very close. In comparison with study by Vieillard-Baron, the R/LVEDA in this study was smaller (interquartile 0.630.72 vs. $0.7-0.9$ ), which might partly explain this result [10]. Next, the CVP is the intramural pressure rather than the transmural pressure of the RV, while the actual pressure that determines RV preload is the CVP relative to the pressure surrounding the heart [19-22]. We cannot exclude conditions where the transmural pressure 


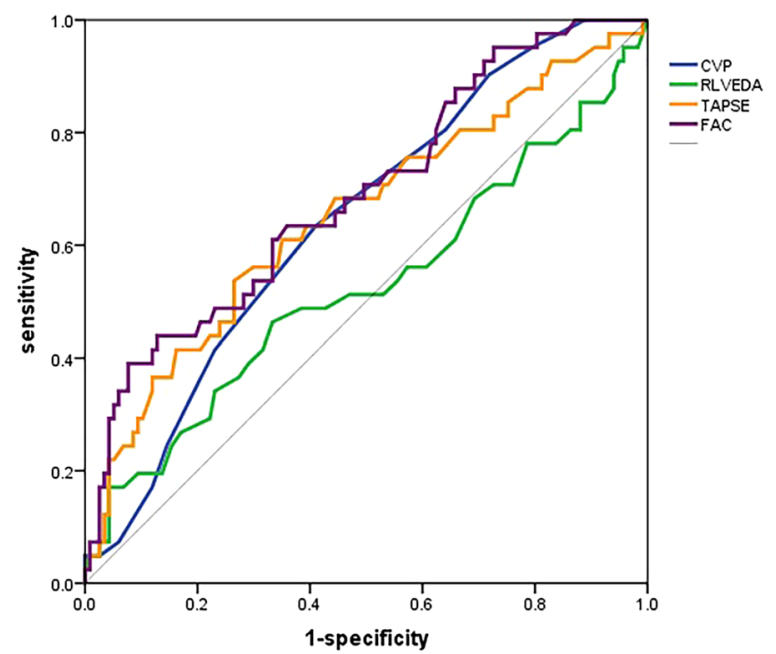

Fig. 3 ROC curve analysis of CVP, R/LVEDA, TAPSE, and FAC for 30 day mortality. CVP central venous pressure, R/LVEDA ratio of right and left ventricular end-diastolic area, TAPSE tricuspid annular plane systolic excursion, FAC fractional area change. The ROC analysis showed that the area under the curve for CVP, R/LVEDA, TAPSE and FAC were $0.644, p=0.006 ; 0.525, p=0.634 ; 0.652, p=0.004$ and $0.690, p<0.001$, respectively

was normal, while CVP increased due to elevated pleural pressure. Furthermore, LV systolic function is often compromised in septic patients, and a concomitant RV dysfunction might ensue, probably because the LV contributes $30 \%$ of the contraction force to RV systolic function [3, 23, 24]. In this case, R/LVEDA might not be enough to diagnose RV enlargement. If the LV is dilated, RV size may be underestimated, and quantification of RV size should be performed independently to determine if there is RV dilation $[18,25]$. Therefore, we suppose that the inherent definition of RVF might result in the overlap of CVP among the four groups, which hopefully would justify the combination of RVF and RVSD in the evaluation of RV.

This study found that RVF alone was not associated with 30-day mortality. Several reasons might help explain this finding. First, the cut-off value of CVP to detect RVF was relatively low. The recommended range of CVP was from 8 to $12 \mathrm{mmHg}$, or even $12-15 \mathrm{mmHg}$ for patients on mechanical ventilation in the SSC guidelines [26]. The interagency Registry for Mechanically Assisted Circulatory Support defines RV failure as an elevated CVP ' $16 \mathrm{mmHg}$ and end-organ dysfunction [27]. Second, we did not notice a significant decrease in the cardiac index of the RVF only group. The patients seemed to be in a state of systemic congestion without compromise of cardiac output. An acute increase in either preload or afterload is immediately associated with RV dilation [28, 29]. No significant difference in TR was found between patients with RVF only and normal patients. Thus, we supposed that the preload (rather than afterload) was responsible for RV dilation in the RVF only group.

RVF and RVSD can occur separately and collectively. A recent study pointed out that RVSD was associated with 28-day mortality in septic patients [17]. However, they did not mention the presence of RVF. It was not clear whether RVSD was still a predictor of mortality if patients with RVF were excluded from their study. This study found that patients with combined RVF-RVSD had the highest mortality. We hypothesized that various types of RV involvement could provide clues about the severity of RV dysfunction (i.e. RVF indicates a lower chance of volume responsiveness, RVSD indicates a higher chance of a decreased cardiac index, and the combination of RVF and RVSD signifies a worse prognosis). Additional research is still warranted in terms of this RV function classification, but we believe it would be clinically relevant.

\section{Limitations}

This study has several limitations. First, given the nature of the retrospective analysis, we did not assess the volume responsiveness of these patients. In future studies, the assessment of volume responsiveness of RVSD patients should be considered and might add value to the classification of RV function. Second, the follow-up was not long enough. We are not certain about the association between RV dysfunction and long-term prognosis. Furthermore, we chose the initial examination to predict outcome when treatment

Table 2 ROC analysis of variables for the prediction of 30-day mortality

\begin{tabular}{lllccrrrr}
\hline Categories & AUC & $\mathbf{9 5 \%} \mathbf{C l}$ & $\boldsymbol{p}$ & Optimum cut-off & Sen & Spe & PPV & NPV \\
\hline CVP (mmHg) & 0.644 & $0.551-0.737$ & 0.006 & 8.5 & 63.4 & 59.0 & 32.0 \\
R/LVEDA & 0.525 & $0.413-0.637$ & 0.634 & - & - & - & - \\
TAPSE (mm) & 0.652 & $0.550-0.754$ & 0.004 & 18.1 & 61.0 & 65.0 & 34.6 \\
FAC (\%) & 0.690 & $0.595-0.786$ & $<0.001$ & 44 & 61.0 & 66.7 & 35.8 & 84.6 \\
\hline
\end{tabular}

CVP central venous pressure, R/LVEDA ratio of right and left end-diastolic area, TAPSE tricuspid annular plane systolic excursion, FAC fractional area change 


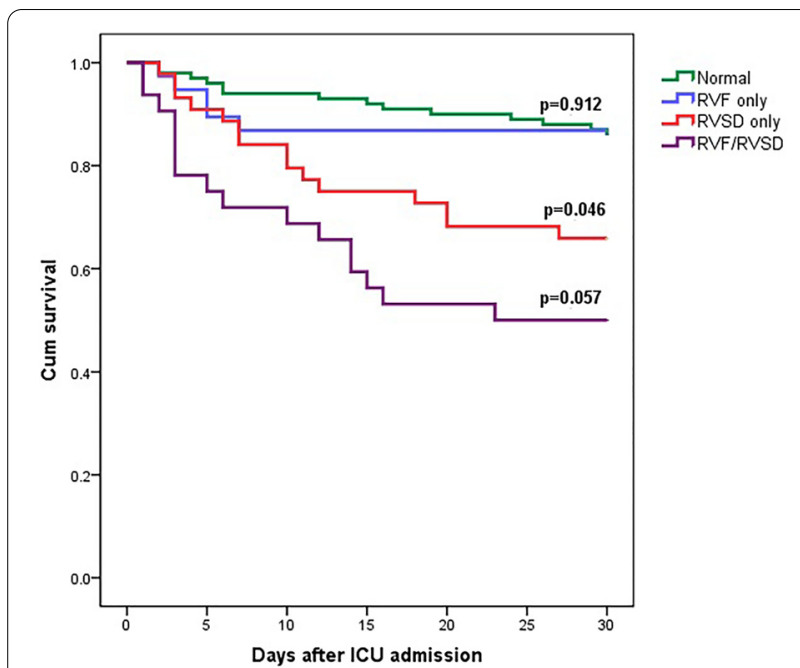

Fig. 4 The Kaplan-Meier curves for estimated survival analysis. The RVF/RVSD group had the highest mortality (RVF/RVSD Vs. RVSD only, log-rank:3.662, $p=0.057$; RVF/RVSD vs. RVF only, log-rank:12.613, $p<0.001 ;$ RVF/RVSD vs. normal, log-rank:25.208, $p<0.001$ ); The RVSD only group had higher mortality than the RVF only and normal groups (RVSD only vs. RVF only, log-rank:3.995, $p=0.046$; RVSD only vs. normal, log-rank: 7.376, $p=0.007)$; No difference was found between the RVF only group and the normal group (RVF only vs. Normal, log-rank: $0.012, p=0.912$ ). RVF only patients with RV failure but without RV systolic dysfunction, RVSD only patients with RVSD but without RV failure, RVF/RVSD patients with combined RVF-RVSD

and response had not occurred. We think a series of echo examinations would yield more robust evidence. Third, RV dilation in combination with septal paradoxical motion can easily assess RV function in a qualitative way [8]. We did not collect information about the septum, which might provide clues about the volume or pressure overload of the RV. Fourth, we only included patients on mechanical ventilation, and the conclusion cannot be applied to spontaneously breathing patients. Last, we had to admit that the terms "RVF only" and "RVSD only" were not perfect. However, we avoided choosing the term "isolated RVF" or "isolated RVSD", which might cause confusion.

\section{Conclusion}

The presence of combined RVF-RVSD was associated with 30-day mortality in mechanically ventilated septic patients. Additional studies are needed to confirm and expand this finding.

\section{Abbreviations}

RV: Right ventricle; LV: Left ventricle; RVF: Right ventricular failure; RVSD: Right ventricular systolic dysfunction; TAPSE: Tricuspid annular plane systolic excursion; FAC: Fractional area change; CVP: Central venous pressure; ICU: Intensive care unit; MV: Mechanical ventilation; LVEF: Left ventricular ejection fraction;
Table 3 Factors associated with 30-day mortality with sensitivity analysis

\begin{tabular}{lllr}
\hline & Hazard ratio & $\mathbf{9 5 \%}$ Cl & p value \\
\hline Univariable analysis & & & \\
Age & 1.006 & $0.989-1.024$ & 0.478 \\
APACHEll & 1.073 & $1.038-1.110$ & $<0.001$ \\
SOFA & 1.230 & $1.134-1.334$ & $<0.001$ \\
PEEP & 1.185 & $1.078-1.302$ & $<0.001$ \\
Pplat & 1.108 & $1.061-1.157$ & $<0.001$ \\
Fluid before echo & 1.002 & $0.991-1.016$ & 0.127 \\
LVEF & 1.001 & $0.980-1.023$ & 0.904 \\
E/e' & 1.063 & $1.013-1.115$ & 0.013 \\
Cl & 0.857 & $0.644-1.142$ & 0.293 \\
RVF only & 0.501 & $0.199-0.263$ & 0.143 \\
RVSD only & 1.815 & $0.991-3.325$ & 0.054 \\
RVF/RVSD & 2.960 & $1.615-5.426$ & $<0.001$ \\
Multivariable analysis & & & \\
SOFA & 1.112 & $1.012-1.223$ & 0.028 \\
Pplat & 1.115 & $1.052-1.182$ & $<0.001$ \\
RVF only & 0.951 & $0.305-2.960$ & 0.931 \\
RVSD only & 1.912 & $0.853-4.287$ & 0.116 \\
RVF/RVSD & 3.004 & $1.370-6.587$ & 0.006 \\
\hline APACHEAcute & & & \\
\hline
\end{tabular}

APACHE Acute Physiology and Chronic Health Evaluation, SOFA Sequential Organ Failure Assessment, CVP central venous pressure, PEEP positive end-expiratory pressure, Pplat plateau pressure, LVEF left ventricular ejection fraction, $\mathrm{Cl}$ cardiac index, RVC only patients with RV congestion but without RV systolic dysfunction, RVSD only patients with RVSD but without RV congestion, RVC/RVSD patients with both RVC and RVSD

TR: Tricuspid regurgitation; R/LVEDA: The ratio of RV end-diastolic area and LV end-diastolic area; LVOT: Left ventricular outflow tract; VTI: Velocity-time integral; APACHE: Acute Physiology and Chronic Health Evaluation; SOFA: Sequential Organ Failure Assessment; HR: Heart rate; MAP: Mean arterial pressure; PEEP: Positive end-expiratory pressure; Pplat: Plateau pressure; Cl: Cardiac index; RVF/RVSD: Presence of combined RVF-RVSD.

\section{Supplementary Information}

The online version contains supplementary material available at https://doi. org/10.1186/s13613-021-00902-9.

Additional file 1: Table S1. General characteristics of all patients. Table S2. Factors associated with 30-day mortality.

\section{Acknowledgements}

This study was performed at Critical Care Department of Peking Union Medical College Hospital. We would like to thank Prof. Chengli Shen, from Division of Surgical Oncology, the Ohio State University Wexner Medical Centre for his kind suggestions on the statistical issue.

\section{Authors' contributions}

$\mathrm{HZ}$ conceived and designed the study, obtained and interpreted data, performed the statistical analysis, and drafted the manuscript. WH analysed data and revised the manuscript. QZ obtained data and revised manuscript. $X C$ revised the manuscript; $X W$ revised the manuscript. DL designed the study and revised the manuscript. All authors read and approved the final manuscript. 


\section{Funding}

Nil.

\section{Availability of data and materials}

All datasets used and analysed during the current study are available from the corresponding author on reasonable request.

\section{Declarations}

\section{Ethics approval and consent to participate}

This study was approved by the ethics committee of Peking Union Medical College Hospital, Beijing, China (Approval No. ZS-1422). Written informed consent was obtained from the next of kin of each patient.

\section{Consent for publication}

Not applicable.

\section{Competing interests}

The authors declare that they have no competing interests.

\section{Author details}

'Department of Critical Care Medicine, Peking Union Medical College Hospital, Chinese Academy of Medical Sciences and Peking Union Medical College, 1\# Shuai Fu Yuan, Dong Cheng District, Beijing 100730, China. ${ }^{2}$ Pittsburgh Heart, Lung, Blood and Vascular Institute, University of Pittsburgh, School of Medicine, Pittsburgh, PA, USA.

Received: 23 January 2021 Accepted: 4 July 2021

Published online: 13 July 2021

\section{References}

1. Martin GS, Mannino DM, Eaton S, Moss M. The epidemiology of sepsis in the United States from 1979 through 2000. N Engl J Med. 2003;348(16):1546-54

2. Gaieski DF, Edwards JM, Kallan MJ, Carr BG. Benchmarking the incidence and mortality of severe sepsis in the United States. Crit Care Med. 2013:41(5):1167-74.

3. Pulido JN, Afessa B, Masaki M, Yuasa T, Gillespie S, Herasevich V, et al. Clinical spectrum, frequency, and significance of myocardial dysfunction in severe sepsis and septic shock. Mayo Clin Proc. 2012;87(7):620-8.

4. Landesberg G, Gilon D, Meroz Y, Georgieva M, Levin PD, Goodman S, et al. Diastolic dysfunction and mortality in severe sepsis and septic shock. Eur Heart J. 2012;33:895-903.

5. Jurcut R, Giusca S, La Gerche A, Vasile S, Ginghina C, Voigt JU. The echocardiographic assessment of the right ventricle: what to do in 2010. Eur J Echocardiogr. 2010;11:81-96.

6. Rudski LG, Lai WW, Afilalo J, Hua L, Handschumacher MD, Chandrasekaran $\mathrm{K}$, et al. Guidelines for the echocardiographic assessment of the right heart in adults: a report from the American Society of Echocardiography endorsed by the European Association of Echocardiography, a registered branch of the European Society of Cardiology, and the Canadian Society of Echocardiography. J Am Soc Echocardiogr. 2010. https://doi.org/10. 1016/j.echo.2010.05.010.

7. Krishnan S, Schmidt GA. Acute right ventricular dysfunction: real-time management with echocardiography. Chest. 2015;147:835-46.

8. Jardin F, Vieillard-Baron A. Monitoring of right-sided heart function. Curr Opin Crit Care. 2005;11:271-9.

9. Vieillard-Baron A. Assessment of right ventricular function. Curr Opin Crit Care. 2009:15(3):254-60.

10. Vieillard-Baron A, Prigent A, Repessé X, Goudelin M, Prat G, Evrard B, et al. Right ventricular failure in septic shock: characterization, incidence and impact on fluid responsiveness. Crit Care. 2020;24:630.

11. Vallabhajosyula S, Kumar M, Pandompatam G, Sakhuja A, Kashyap $\mathrm{R}$, Kashani K, et al. Prognostic impact of isolated right ventricular dysfunction in sepsis and septic shock: an 8-year historical cohort study Ann Intensive Care. 2017;7:94.

12. Zhang H, Lian H, Zhang Q, Chen X, Wang X, Liu D. Prognostic implications of tricuspid annular plane systolic excursion/pulmonary arterial systolic pressure ratio in septic shock patients. Cardiovasc Ultrasound. 2020;18:20.

13. Winkelhorst JC, Bootsma IT, Koetsier PM, de Lange F, Boerma EC. Right ventricular function and long-term outcome in sepsis: a retrospective cohort study. Shock. 2019. https://doi.org/10.1097/SHK.0000000000 001413.

14. Singer M, Deutschman CS, Seymour CW, Shankar-Hari M, Annane D, Bauer $\mathrm{M}$, et al. The Third International Consensus definitions for sepsis and septic shock (Sepsis-3). JAMA. 2016;315:801-10,

15. Sanfilippo F, Huang S, Herpain A, Balik M, Chew MS, Clau-Terré F, et al. The PRICES statement: an ESICM expert consensus on methodology for conducting and reporting critical care echocardiography research studies. Intensive Care Med. 2021;47(1):1-13.

16. Quiñones MA, Otto CM, Stoddard M, Waggoner A, Zoghbi WA, Doppler Quantification Task Force of the Nomenclature and Standards Committee of the American Society of Echocardiography. Recommendations for quantification of Doppler echocardiography: a report from the Doppler Quantification Task Force of the Nomenclature and Standards Committee of the American Society of Echocardiography. J Am Soc Echocardiogr. 2002;15(2):167-84.

17. Lanspa MJ, Cirulis MM, Wiley BM, Olsen TD, Wilson EL, Beesley SM, et al. Right ventricular dysfunction in early sepsis and septic shock. Chest. 2020. https://doi.org/10.1016/j.chest.2020.09.274.

18. Vieillard-Baron A, Naeije R, Haddad F, Bogaard HJ, Bull TM, Fletcher N, et al. Diagnostic workup, etiologies and management of acute right ventricle failure: a state-of-the-art paper. Intensive Care Med. 2018;44:774-90.

19. Magder S. Central venous pressure: a useful but not so simple measurement. Crit Care Med. 2006:34:2224-7.

20. Pinsky MR. My paper 20 years later: effect of positive end-expiratory pressure on right ventricular function in humans. Intensive Care Med. 2014;40:935-41.

21. Pinsky MR, Desmet JM, Vincent JL. Effect of positive end-expiratory pressure on right ventricular function in humans. Am Rev Respir Dis. 1992;146:681-7.

22. Gelman S. Venous function and central venous pressure: a physiologic story. Anesthesiology. 2008;108(4):735-48.

23. Santamore WP, Dell'Italia LJ. Ventricular interdependence: significant left ventricular contributions to right ventricular systolic function. Prog Cardiovasc Dis. 1998:40:289-308.

24. Kjaergaard J, Iversen KK, Akkan D, Møller JE, Køber LV, Torp-Pedersen C, et al. Predictors of right ventricular function as measured by tricuspid annular plane systolic excursion in heart failure. Cardiovasc Ultrasound. 2009;7:51.

25. Orde S, Slama M, Hilton A, Yastrebov K, McLean A. Pearls and pitfalls in comprehensive critical care echocardiography. Crit Care. 2017;21(1):279.

26. Dellinger RP, Levy MM, Carlet JM, Bion J, Parker MM, Jaeschke R, et al. Surviving Sepsis Campaign: international guidelines for management of severe sepsis and septic shock: 2008. Intensive Care Med. 2008;34:17-60.

27. Amsallem M, Mercier O, Kobayashi Y, Moneghetti K, Haddad F. Forgotten no more: a focused update on the right ventricle in cardiovascular disease. JACC Heart Fail. 2018;6:891-903.

28. Sanz J, Sánchez-Quintana D, Bossone E, Bogaard HJ, Naeije R. Anatomy, function, and dysfunction of the right ventricle: JACC state-of-the-art review. J Am Coll Cardiol. 2019;73:1463-82.

29. Harjola VP, Mebazaa A, Čelutkiené J, Bettex D, Bueno H, Chioncel O, et al. Contemporary management of acute right ventricular failure: a statement from the Heart Failure Association and the Working Group on Pulmonary Circulation and Right Ventricular Function of the European Society of Cardiology. Eur J Heart Fail. 2016;18:226-41.

\section{Publisher's Note}

Springer Nature remains neutral with regard to jurisdictional claims in published maps and institutional affiliations. 